\title{
Leafwise hyperbolicity; a correction
}

\section{John Cantwell* and Lawrence Conlon**}

In [1], a proof of the following theorem was proposed.

THEOREM 1. Let $(M, \mathscr{F})$ be a $C^{2}$-foliated manifold of codimension 1 , transversely orientable and such that $M$ is compact, every leaf is proper, and $\mathscr{F}$ is tangent to $\partial M$. If no leaf of $\mathscr{F}$ is a torus or a sphere, then there is a Riemannian metric on $M$ relative to which each leaf of $\mathscr{F}$ has constant curvature -1 .

This theorem is correct, but there was an erroneous step in the proof, namely [1, Lemma (2.2)]. We are grateful to S. Matsumoto and N. Tsuchiya for pointing this out to us.

We fix the hypotheses of Theorem 1. A metric $g$ with the property in that theorem will be called leafwise hyperbolic.

Let $M_{0} \subseteq M_{1} \subseteq \cdots \subseteq M_{k} \subseteq M_{k+1} \subseteq \cdots$ denote the level filtration [2]. Each $M_{k}$ is a compact, nonempty, $\mathscr{F}$-saturated set, the leaves in $M_{k} \backslash M_{k-1}$ being the leaves of $\mathscr{F}$ at level $k$. When all leaves are proper, it has become customary to use the term "depth" rather than "level". Since all leaves are proper and the foliation is of class $C^{2}$, every leaf of $\mathscr{F}$ has finite depth, hence $M=\bigcup_{k=0}^{\infty} M_{k}$.

PROPOSITION 1. Let $M_{k}$ denote the union of leaves at dephts at most $k$. Then there is a nest $W_{0} \subseteq W_{1} \subseteq \cdots \subseteq W_{k} \subseteq W_{k+1} \subseteq \cdots \subseteq M$, where $W_{k}$ is an open neighborhood of $M_{k}$, and there is a Riemannian metric $g_{k}$ on $M$ such that $g_{k} \mid W_{k}$ is leafwise hyperbolic for $\mathscr{F} \mid W_{k}, \forall k \geq 0$.

Theorem 1 follows. Indeed, $\left\{W_{k}\right\}_{k=0}^{\infty}$ is an open, nested cover of the compact manifold $M$, hence passing to a finite subcover yields a value of $k$ for which $W_{k}=M$. It remains, then, to prove Proposition 1 .

We fix a smooth, 1-dimensional foliation $\mathscr{F}^{\perp}$, everywhere transverse to $\mathscr{F}$. Projections along the leaves of $\mathscr{F}^{\perp}$ can be used to define local diffeomorphisms between leaves of $\mathscr{F}$.

\footnotetext{
* Research partially supported by N.S.F. Contract DMS-8900127

** Reséarch partially supported by N.S.F. Contract DMS-8822462
} 
If $U \subseteq M$ is an open, connected, $\mathscr{F}$-saturated set, we use the notations $\hat{U}, \hat{\imath}: \hat{U} \rightarrow M, \hat{F}=\hat{\imath}^{-1}(\mathscr{F})$, and $\hat{\mathscr{F}}^{\perp}=\hat{\imath}^{-1}\left(\mathscr{F}^{\perp}\right)$ from [1], [2], et al., for the completion of $U$, its natural immersion into $M$, and the induced foliations of $\hat{U}$, respectively. Recall that $U$ and $\hat{U}$ are called foliated products if $\hat{U}$ is diffeomorphic to $L \times[0,1]$ in such a way that the leaves of $\hat{F}^{\perp}$ are the [0,1]-fibers. Recall that, if $U$ is a foliated product, then $\hat{\imath}(\partial \hat{U})$ is either a single leaf or a pair of leaves of $\mathscr{F}$.

DEFINITION 1. A closed subset $X \subset M$ that is a finite union of leaves of will be called a skeleton if each component of $M \backslash X$ is a foliated product. If $k$ is the highest depth of the leaves in $X$, the skeleton has depth $k$. We will say that $X$ (of depth $k$ ) is a full skeleton if, for each component $U$ of $M \backslash X$, at least one of the following holds.

(1) Every leaf $L$ of $\hat{F}$ has image $\hat{\imath}(L)$ at the same depth $k_{0} \leq k$.

(2) If $L \subset \partial \hat{U}$ is a boundary leaf, then $\hat{\imath}(L)$ is a leaf at depth $k$.

If $X$ is a skeleton, it was proven in [1, (1.2)] that there is an open neighborhood $W \supset X$ and a Riemannian metric $g$ on $M$ such that $g \mid W$ is leafwise hyperbolic for $\mathscr{F} \mid W$. Furthermore, projection along the leaves of $\mathscr{F}^{\perp}$ defines local isometries between the leaves of $\mathscr{F} \mid W$. Finally, $\widehat{U} \backslash \hat{\imath}^{-1}(W)$ is compact, for each component $U$ of $M \backslash X$.

LEMMA 1. If there is a full skeleton $X$ of depth $N$, then there is a neighborhood $W_{N} \supset M_{N}$ and a Riemannian metric $g_{N}$ on $M$ which is leafwise hyperbolic on $W_{N}$.

Proof. Let $U$ be a component of $M \backslash X$. There are two cases, corresponding to possibilities (1) and (2) of Definition 1.

(1) In this case, the proof of [1, Lemma (2.1)] shows how to extend the metric smoothly over all of $U$ so as to make the curvature of the leaves of $\mathscr{F} \mid U$ constantly -1 . Indeed, the metric was already appropriately defined on all but a compact submanifold $A \times[0,1] \subset \hat{U}$ and $F$ induces the product foliation on this submanifold. A deformation argument, using the Teichmüller space of $A$, created the extension. (The error in [1] was to claim that, even in the second case, where the foliation of $A \times[0,1]$ was not a product, the above metric on the product could be "tilted" to give a hyperbolic metric along the leaves.)

(2) We assume that the situation in (1) does not also occur. In this case, the argument is actually easier. Since $M_{N}$ is compact $[2,(4.6)], \hat{\imath}^{-1}\left(M_{N}\right) \cap \hat{U}=L \times C$, where $C \subset[0,1]$ is a closed subset containing $\{0,1\}$. Since $U \backslash M_{N} \neq \varnothing,[0,1] \backslash C$ has at least one component $(a, b)$. Let $a<a^{\prime}<b^{\prime}<b$. The metric $g$ is already defined on $W \cap \hat{\imath}(\hat{U})$ in such a way that projections along $\mathscr{F}^{\perp}$ are local isometries between leaves. Using the projections $p^{+}: L \times\left(b^{\prime}, 1\right] \rightarrow L \times\{1\}$ and $p^{-}: L \times\left[0, a^{\prime}\right) \rightarrow$ 
$L \times\{0\}$, one lifts this metric smoothly to $L \times\left[0, a^{\prime}\right) \cup L \times\left(b^{\prime}, 1\right]$. This metric agrees with $g$ wherever both are defined.

Finite repetition of this argument, as $U$ ranges over the components of $M \backslash X$, completes the proof.

LEMMA 2. For some integer $N \geq 0$, there exists a full skeleton of depth $N$.

Proof. As in $[1,(1.1)]$, one constructs a skeleton $X$. Let $N$ be the depth of $X$. If $X$ is not full, consider a component $U$ of $M \backslash X$ with boundary component(s) at depth $k<N$. If every leaf of $\mathscr{F} \mid U$ is at depth $k$, there is nothing to do. Otherwise, there is a leaf $L \subset U$ at depth $k+1 \leq N$. It is elementary that $X^{\prime}=X \cup L$ is again a skeleton of depth $N$. If $X^{\prime}$ is not full repeat the process for $X^{\prime}$. Finite repetition will ultimately produce a full skeleton of depth $N$.

For $0 \leq k \leq N$, we set $W_{k}=W_{N}$ and $g_{k}=g_{N}$. We also set $X=X_{N}$.

Each component $U_{i}$ of $M \backslash X_{N}$ that has not been engulfed by $W_{N}$ must contain a leaf $L_{i}$ at depth $N+1$. Throwing these finitely many leaves in with $X_{N}$ provides a full skeleton $X_{N+1}$ of depth $N+1$. An application of Lemma 1 produces $W_{N+1}$ and $g_{N+1}$ as desired. It is not hard to see that $W_{N+1}$ can be chosen to engulf $W_{N}$. Proceeding in this way, we construct the nest of open sets and the metrics as in Proposition 1.

REMARK. Projection along the leaves of $\mathscr{F} \perp$ does not always define local isometries between the leaves of $\mathscr{F}$. In the pieces $A \times[0,1]$, where the metric is extended by a deformation in Teichmüller space, these projections will not be isometric. If it were possible to avoid introducing these regions, it would follow that the leafwise hyperbolic metric for $\mathscr{F}$ is a bundlelike metric for $\mathscr{F}^{\perp}$, hence that the leaves of $\mathscr{F}$ are totally geodesic in this metric. But totally geodesic foliations of compact 3-manifolds by surfaces are relatively rare.

\section{REFERENCES}

[1] Cantwell J. and Conlon L. Leafwise hyperbolicity of proper foliations. Comment. Math. Helv., 64 (1989) 329-337.

[2] Cantwell J. and CONlon L. Poincaré-Bendixson theory for leaves of codimension one. Trans. Amer. Math. Soc., 265 (1981) $181-209$.

\section{St. Louis University}

Department of Mathematics

Ritter Hall

St. Louis, MO 63103, USA
Washington University

Department of Mathematics

Campus Box 1146

St. Louis, MO 63130-4899, USA

Received October 20, 1990 